\title{
Transfection of the mutant MYH9 cDNA reproduces the most typical cellular phenotype of MYH9-related disease in different cell
} lines

\author{
Emanuele Panza ${ }^{1}$, Monica Marini², Alessandro Pecci ${ }^{3}$, \\ Francesca Giacopelli2,4, Valeria Bozzi ${ }^{3}$, Marco Seri ${ }^{1}$, Carlo Balduini ${ }^{3}$ and \\ Roberto Ravazzolo*2,4
}

Address: ${ }^{1}$ Medical Genetics Unit, Department of Gynecology, Obstetrics and Pediatrics, University of Bologna, Bologna, Italy, ${ }^{2}$ Laboratory of Molecular Genetics, G. Gaslini Institute, Genova, Italy, ${ }^{3}$ Department of Internal Medicine, IRCCS Policlinico S. Matteo, University of Pavia, Pavia, Italy and ${ }^{4}$ Department of Pediatrics and CEBR, University of Genova, Genova, Italy

Email: Emanuele Panza - emanuele.panza@unibo.it; Monica Marini - monica.marini@unige.it; Alessandro Pecci - alessandro.pecci@unipv.it; Francesca Giacopelli - fgiacopelli@yahoo.com; Valeria Bozzi - bozzivaleria@libero.it; Marco Seri - marco.seri@unibo.it;

Carlo Balduini - c.balduini@smatteo.pv.it; Roberto Ravazzolo* - rravazzo@unige.it

* Corresponding author

Published: I December 2008

PathoGenetics 2008, I:5 doi:10.1186/1755-8417-1-5

This article is available from: http://www.pathogeneticsjournal.com/content/I/1/5

(C) 2008 Panza et al; licensee BioMed Central Ltd.

This is an Open Access article distributed under the terms of the Creative Commons Attribution License (http://creativecommons.org/licenses/by/2.0), which permits unrestricted use, distribution, and reproduction in any medium, provided the original work is properly cited.
Received: 30 May 2008

Accepted: I December 2008

\begin{abstract}
Background: Heterozygous mutations of MYH9, encoding the Non-Muscular Myosin Heavy Chain-IIA (NMMHC-IIA), cause a complex disorder named MYH9-related disease, characterized by a combination of different phenotypic features. At birth, patients present platelet macrocytosis, thrombocytopenia and leukocyte inclusions containing NMMHC-IIA. Moreover, later in life some of them develop the additional features of sensorineural hearing loss, cataracts and/or glomerulonephritis that sometimes leads to end stage renal failure.

Results: To clarify the mechanism by which the mutant NMMHC-IIA could cause phenotypic anomalies at the cellular level, we examined the effect of transfection of the full-length mutated D I424H MYH9 cDNAs. We have observed, by confocal microscopy, abnormal distribution of the protein and formation of rod-like aggregates reminiscent of the leukocyte inclusions found in patients. Co-transfection of differently labeled wild-type and mutant full-length cDNAs showed the simultaneous presence of both forms of the protein in the intracellular aggregates.
\end{abstract}

Conclusion: These findings suggest that the NMMHC-IIA mutated in position I424 is able to interact with the WT form in living cells, despite part of the mutant protein precipitates in nonfunctional aggregates. Transfection of the entire WT or mutant MYH9 in cell lines represents a powerful experimental model to investigate consequences of $\mathrm{MYH9}$ mutations.

\section{Background}

Heterozygous mutations in the MYH9 gene, encoding the Non-Muscle Myosin Heavy Chain IIA (NMMHC-IIA), are responsible for the recently defined MYH9-related disease
(MYH9-RD) [1]. This entity includes clinical phenotypes previously classified as distinct disorders: May-Hegglin anomaly, Sebastian syndrome, Fechtner syndrome, and Epstein syndrome. All patients present since birth throm- 
bocytopenia and leukocyte inclusions consisting of aggregates of NMMHC-IIA. However, during infancy or adult life many subjects develop the additional features of sensorineural hearing loss, cataracts, and/or progressive nephropathy leading to renal failure [1].

NMMHC-IIA is a conventional, non-sarcomeric myosin expressed in most cells and tissues, where it is involved in several functions including cytokinesis, cell motility, and maintenance of cell shape [2]. The N-terminal portion of NMMHC-IIA forms the myosin globular head, responsible for ATPase and actin-binding activity, while the C-terminal tail region regulates both dimerization of heavy chains in coiled-coil structures and association of myosin molecules into functional filaments [2].

The mechanisms by which MYH9 mutations cause MYH9$\mathrm{RD}$ are poorly defined, and both haploinsufficiency and a dominant-negative effect of the mutated protein have been hypothesized [3-6]. In particular, studies on NMMHC-IIA tail fragments demonstrated that differently mutated proteins could interact to a different extent with WT counterparts to exert a dominant-negative biochemical effect [5]. Nevertheless, investigations on megakaryocytes and platelets from patients with MYH9-RD suggested that mutant NMMHC-IIA proteins could not be expressed in living cells, possibly because of their high instability $[3,6]$. Difficulties in addressing these issues include the unavailability of the entire NMMHC-IIA [7], so that experimental approaches were so far limited to in vitro studies on either $\mathrm{N}$-terminal or $\mathrm{C}$-terminal portions of the molecule [5].

Here we present the results of transfection with the entire NMMHC-IIA molecule both in wild-type (WT) and mutated form (D1424H) in COS-7 and HeLa cell lines.

\section{Methods}

The full-length 5883 bp MYH9 cDNA was cloned using the Gateway system (Invitrogen). RNA was extracted from a lymphoblastoid cell line from a control individual. Five hundred nanograms of total RNA were specifically retrotranscribed in order to obtain MYH9 cDNA (Transcriptor First Strand cDNA Synthesis Kit, Roche). The resulting cDNA was used for PCR amplification. Primers, which included the attB1 and attB2 recombination sites, were:

Forward-AttB1: GGGGACAAGTTTGTACAAAAAAGCAGGCTCCATGGCACAGCAAGCTGCC;

Reverse-AttB2: GGGGACCACTTTGTACAAGAAAGCTGGGTCTTATTCGGCAGGTTTGGCC.

Forty nanograms of cDNA were used to amplify the entire MYH9 coding sequence.
The PCR fragment was introduced into plasmid vector pDONOR207 (Invitrogen) via the BP recombination reaction, according to the recommendations of the manufacturer, to generate the entry clone. Control of the resulting cDNA was performed by complete sequencing. Comparison of the sequence in our clone with the cDNA reported in the NCBI database (NM_002473) did not reveal any difference.

Site directed mutagenesis was performed using a commercial kit (Quick Change, Stratagene) to introduce a G to C transversion in position 4270 , in order to obtain the D1424H mutant. WT and D1424H constructs were then used to perform LR recombination reactions with the Gateway system compatible expression vector pcDNA3.1/ nV5-DEST (Invitrogen) and Gateway converted Flag vector to express N-terminally tagged V5, or Flag fusion proteins, respectively. The integrity of all constructs was confirmed by direct complete DNA sequencing.

COS-7 and HeLa cells were maintained in Dulbecco's modified Eagle's medium supplemented with $10 \%(\mathrm{v} / \mathrm{v})$ fetal bovine serum (GibcoBRL), $2 \mathrm{mM}$ L-glutamine, 100 $\mathrm{U} / \mathrm{ml}$ penicillin, $100 \mu \mathrm{g} / \mathrm{ml}$ streptomycin at $37^{\circ} \mathrm{C}$ in a humidified atmosphere with 5\% CO2. Transfections of both cell lines were performed using the Polyfect reagent (Quiagen), according to the manufacturer's instructions, on cells plated at $50-60 \%$ confluence and transfected at an estimated $80-90 \%$ confluence after $24 \mathrm{~h}$. Cells were harvested 24, 48 and 72 hours after transfection and cytospun onto slides for immunofluorescence staining.

Standard peripheral blood smears were prepared from two patients with MYH9-RD caused by D1424H substitution of NMMHC-IIA. Both patients have been already reported [1]. The institutional review board of Fondazione IRCCS Policlinico San Matteo, University of Pavia, Italy, approved the study and patients gave written informed consent to the study.

The following primary antibodies (Abs) were used: rabbit polyclonal PR-B440P (Covance Research Products Berkeley, CA) recognizing the 12-residues sequence (GKADGAEAKPAE) of the C-terminus of NMMHC-IIA [8], mouse monoclonal NMG2 to a specific epitope of the N-terminus of NMMHC-IIA (a kind gift of Dr Saverio Sartore, Biomedical Sciences, University of Padua, Italy) [9]; rabbit polyclonal anti-flag (Sigma, St. Louis, MI), and mouse monoclonal anti-V5 (Invitrogen).

Secondary antibodies were goat anti-mouse or anti-rabbit conjugated with either Alexa Fluor 488 or Alexa Fluor 594 (Invitrogen). Confocal microscopy was performed through the TCS SPII confocal laser scanning microscopy system (Leica, Heidelberg, Germany), equipped with a 
Leica DM IRBE inverted microscope, as previously described [6]. Confocal optical sections were performed every $500 \mathrm{~nm}$. Conventional fluorescence microscopy was performed through an Axioscope 2 Plus microscope (Carl Zeiss, Gottingen, Germany), as described [6].

\section{Results and discussion}

The entire 5883 bp MYH9 cDNAs, both WT and D1424H form, have been transfected in COS-7 or in HeLa cell lines. Immunofluorescence staining showed that COS-7 cells, which constitutively do not express MYH9, expressed the transfected WT or D1424H NMMHC-IIAs, and that the signal of these molecules was stable for at least 72 hours after transfection. No NMMHC-IIA signal has been observed in non-transfected cells or in cells transfected with the vector alone. Transfected COS-7 cells were similarly labeled by antibodies specific either to the N-terminus or to the C-terminus of the protein, demonstrating that the entire WT or mutant NMMHC-IIA were expressed.

Confocal microscopy demonstrated that the signal of the respective protein tags (V5 for WT, flag for D1424H) was completely superimposable to the NMMHC-IIA signal, confirming that staining for the tags was reliable to assess both expression and intracellular distribution of the respective exogenous NMMHC-IIAs (data not shown). While in COS-7 transfected with WT MYH9 the NMMHCIIA signal was uniformly distributed in the cytoplasm, in cells transfected with the D $1424 \mathrm{H}$ construct NMMHC-IIA formed rod-like protein aggregates similar to those
WILD-TYPE NMMHC-IIA

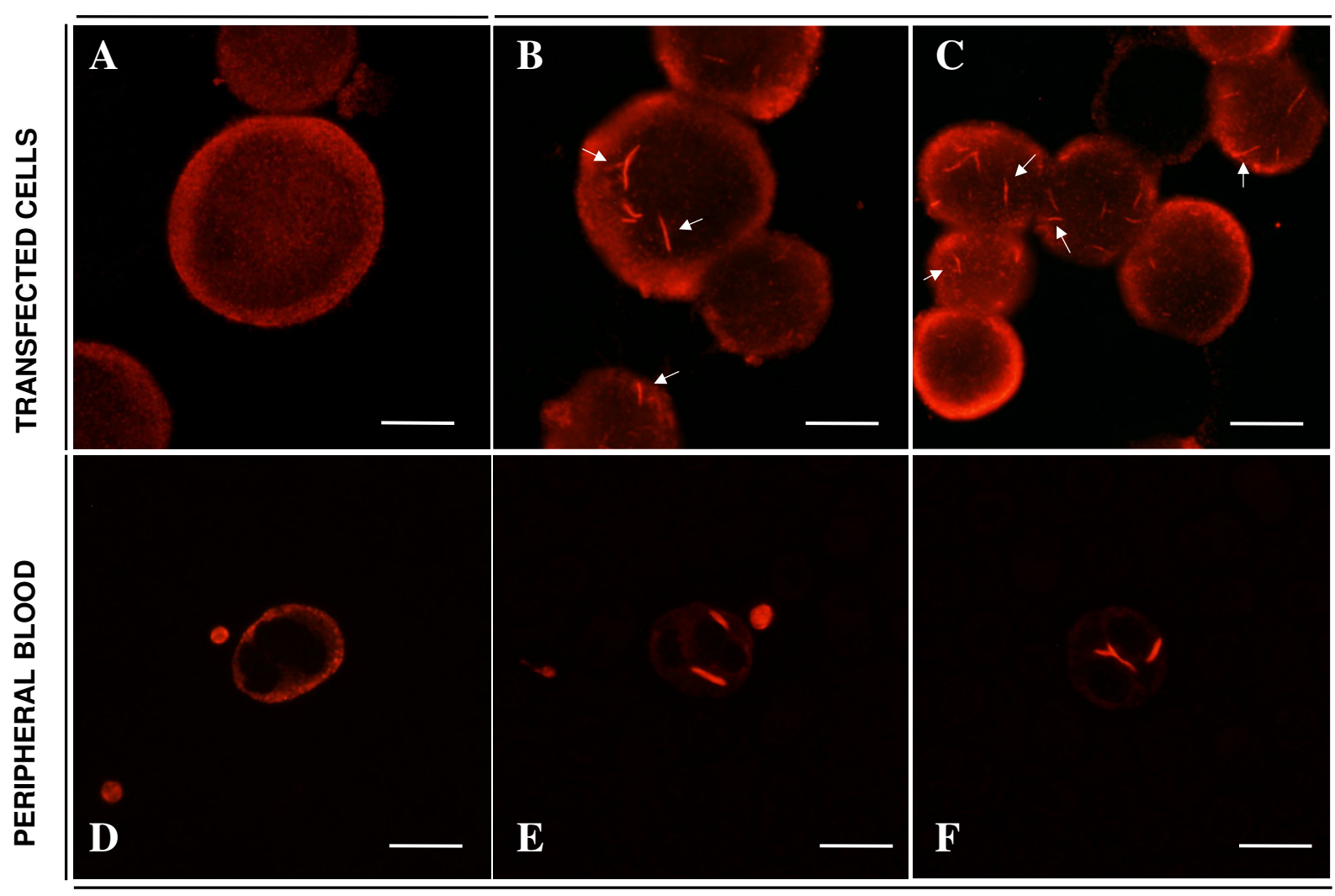

Figure I

Transfection of D I424H MYH9 in COS-7 or HeLa cells results in the formation of NMMHC-IIA aggregates similar to those of granulocytes of MYH9-RD patients carrying the same mutation. A) COS-7 cells transfected with WT MYH9 cDNA. B) COS-7 cells transfected with DI424H MYH9 cDNA. C) HeLa cells transfected with DI424H MYH9 cDNA. D) Staining of granulocyte from an unaffected individual. E) and F) Staining of granulocytes from patients with the DI424H substitution obtained by labeling the peripheral blood smears for NMMHC-IIA. Scale bars correspond to I0 $\mu$ m. Arrows indicate rod-like aggregates. 
observable in granulocytes of patients carrying the D1424H substitution (Figure 1A and 1B). To show such similarity, peripheral blood smears were prepared from two patients with MYH9-RD caused by the D1424H substitution of NMMHC-IIA, compared with an unaffected individual (Figure 1D-F). Both patients had already been reported [1].

These aggregates of mutant NMMHC-IIA were present in most transfected cells, and they were usually evident over a diffuse cytoplasmic staining. When we transfected with the same constructs HeLa cells, which constitutively express NMMHC-IIA, we obtained the same results as in COS-7 (Figure 1C).

Thus, transfection of the entire MYH9 mutant cDNA reproduced in two different cell lines, independently from their endogenous myosin expression, the NMMHC-IIA aggregates that are typical of granulocytic cells in MYH9$\mathrm{RD}[1,4,10]$. Interestingly, a similar distribution of NMMHC-IIA was observed in tubular epithelia cells from the only patient with MYH9-RD whose renal biopsy was investigated by immunohystochemistry [10], suggesting that this behavior of mutant NMMHC-IIA could be common to different cell types in vivo.
Since in cells of MYH9-RD patients both a WT and a mutant allele are present, we co-transfected COS-7 or HeLa cells with both WT and D1424H cDNAs and investigated intracellular localization of the two exogenous NMMHC-IIAs by double-labeling cells for the respective protein tags. In co-transfected cells the mutant NMMHCIIA showed a distribution similar to that observed in cells transfected with mutant MYH9 alone, with part of the flag signal diffused in the cytoplasm and part organized in rod-like aggregates (Figure $2 \mathrm{~A}$ ). We noticed that aggregates were less frequent than in cells transfected with D1424H cDNA alone. Confocal analysis demonstrated that the V5 (WT) signal presented a high degree of colocalization with the flag (mutant) signal diffused in the cytoplasm, thus suggesting that transfected WT and D1424H molecules interacted in these cells (Figure 2AC). Interestingly, the V5 signal was detected also at the level of some of the aggregates disclosed by the flag reaction, although it was less intense than the mutant signal (Figure 2A-C). This finding indicates that rod-like aggregates were mainly constituted by D1424H NMMHC-IIA, although WT protein was trapped into some of these structures. This observation is consistent with results previously obtained in granulocytes of patients with non-

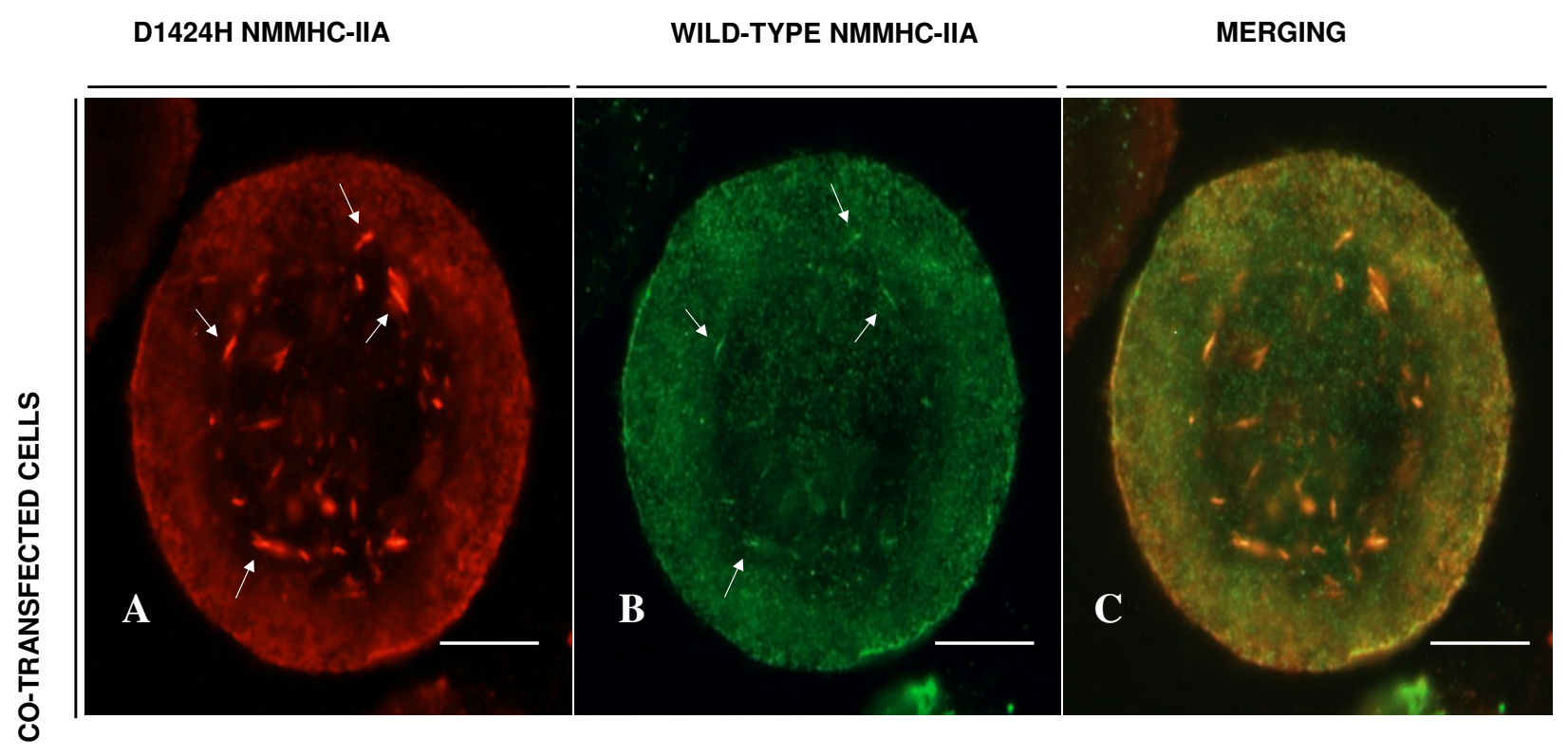

\section{Figure 2}

Exogenous DI 424H and WT NMMHC-IIA co-localize in co-transfected COS-7 cells. A) COS-7 cells co-transfected with both DI424H and WT MYH9 cDNAs tagged at the N-terminal end with flag and V5 epitopes, respectively: red staining for the flag epitope (mutant). B) Same co-transfected cells for the V5 epitope (WT, green). C) Merging of images resulting from overlapping of the two channels. Scale bars correspond to $10 \mu \mathrm{m}$. Arrows indicate rod-like aggregates. 
sense or frameshift MYH9 mutations resulting in a truncated NMMHC-IIA $[4,6,11]$.

Results of cotransfection similar to those in COS-7 cells were also obtained in HeLa cells (not shown).

\section{Conclusion}

These findings suggest that the NMMHC-IIA mutated in position 1424 is able to interact with the WT form in living cells, despite part of the mutant protein precipitates in non-functional aggregates. This observation is consistent with previous studies on an in vitro model, which demonstrated that the D1424N C-terminal fragment of NMMHC-IIA, when mixed with its WT counterpart, is able to form paracrystalline assemblies similar to those of the WT/WT interaction [5]. The fact that NMMHC-IIA mutated in position 1424 retains the ability to interact with WT protein represents the basis for it to exert a dominant-negative biochemical effect on the normal protein.

Transfection of the entire WT or mutant MYH9 in cell lines represents a promising experimental model to investigate consequences of MYH9 mutations in cells.

\section{Competing interests}

The authors declare that they have no competing interests.

\section{Authors' contributions}

RR proposed and supervised the study, analyzed data, and co-wrote the article; EP prepared the full-length cDNA construct and performed site specific mutagenesis; MM carried out transfection experiments; AP performed confocal microscopy analysis; FG collaborated in transfection experiments; VB collaborated in confocal microscopy analysis; MS supervised construct preparation, provided intellectual expertise, and co-wrote the article; CB supervised morphological study, provided intellectual expertise, and co-wrote the article. EP, MM and AP contributed equally to the work.

\section{Acknowledgements}

The research was supported by a grant for Rare Diseases from the Istituto Superiore di Sanità, Ministry of Health, Italy.

\section{References}

I. Seri M, Pecci A, Di Bari F, Cusano R, Savino M, Panza E, Nigro A, Noris P, Gangarossa S, Rocca B, Gresele P, Bizzaro N, Malatesta P, Koivisto PA, Longo I, Musso R, Pecoraro C, lolascon A, Magrini U, Rodriguez Soriano J, Renieri A, Ghiggeri GM, Ravazzolo R, Balduini CL, Savoia A: MYH9-related disease: May-Hegglin anomaly, Sebastian syndrome, Fechtner syndrome, and Epstein syndrome are not distinct entities but represent a variable expression of a single illness. Medicine (Baltimore) 2003, 82:203-2I5.

2. Sellers JR: Myosins: a diverse superfamily. Biochim Biophys Acta 2000, 1496:3-22.

3. Deutsch S, Rideau A, Bochaton-Piallat ML, Merla G, Geinoz A, Gabbiani G, Schwede T, Matthes T, Antonarakis SE, Beris P: Asp 1424Asn MYH9 mutation results in an unstable protein responsible for the phenotypes in May-Hegglin anomalyl Fechtner syndrome. Blood 2003, 102:529-534.

4. Kunishima S, Matsushita T, Kojima T, Sako M, Kimura F, Jo EK, Inoue $C$, Kamiya T, Saito H: Immunofluorescence analysis of neutrophil nonmuscle myosin heavy chain-A in MYH9 disorders: association of subcellular localization with MYH9 mutations. Lab Invest 2003, 83: I I5-I22.

5. Franke JD, Dong F, Rickoll WL, Kelley MJ, Kiehart DP: Rod mutations associated with MYH9-related disorders disrupt nonmuscle myosin-IIA assembly. Blood 2005, 105:161-169.

6. Pecci A, Canobbio I, Balduini A, Stefanini L, Cisterna B, Marseglia C, Noris P, Savoia A, Balduini CL, Torti M: Pathogenetic mechanisms of hematological abnormalities of patients with MYH9 mutations. Hum Mol Genet 2005, 14:3169-3I78.

7. Takubo T, Wakui S, Daigo K, Kurokata K, Ohashi T, Katayama K, Hino $M$ : Expression of non-muscle type myosin heavy polypeptide 9 (MYH9) in mammalian cells. Eur J Histochem 2003, 47:345-352.

8. Maupin P, Phillips CL, Adelstein RS, Pollard TD: Differential localization of myosin-II isozymes in human cultured cells and blood cells. J Cell Sci 1994, I07(Pt I I):3077-3090.

9. Pecci A, Noris P, Invernizzi R, Savoia A, Seri M, Ghiggeri GM, Sartore $S$, Gangarossa S, Bizzaro N, Balduini CL: Immunocytochemistry for the heavy chain of the non-muscle myosin IIA as a diagnostic tool for MYH9-related disorders. Br J Haematol 2002, I I 7:164-I67.

10. Ghiggeri GM, Caridi G, Magrini U, Sessa A, Savoia A, Seri M, Pecci A, Romagnoli R, Gangarossa S, Noris P, Sartore S, Necchi V, Ravazzolo $R$, Balduini CL: Genetics, clinical and pathological features of glomerulonephritis associated with mutations of nonmuscle myosin IIA (Fechtner syndrome). Am J Kidney Dis 2003, $41: 95-104$.

II. Kunishima S, Hamaguchi M, Saito H: Differential expression of wild-type and mutant NMMHC-IIA polypeptides in blood cells suggests cell-specific regulation mechanisms in MYH9 disorders. Blood 2008, I I 1:3015-3023.

Publish with Bio Med Central and every scientist can read your work free of charge

"BioMed Central will be the most significant development for disseminating the results of biomedical research in our lifetime. "

Sir Paul Nurse, Cancer Research UK

Your research papers will be:

- available free of charge to the entire biomedical community

- peer reviewed and published immediately upon acceptance

- cited in PubMed and archived on PubMed Central

- yours - you keep the copyright 